\title{
Arbitrary reference, numbers, and propositions
}

\author{
Michele Palmira \\ michelepalmira@gmail.com \\ University of Barcelona and LOGOS Research Group \\ Forthcoming in the European Journal of Philosophy, online first, \\ DOI: $10.1111 /$ ejop.12367 \\ Penultimate Draft \\ Please refer to the published version
}

\begin{abstract}
Reductionist realist accounts of certain entities, such as the natural numbers and propositions, have been taken to be fatally undermined by what we may call the problem of arbitrary identification. The problem is that there are multiple and equally adequate reductions of the natural numbers to sets (see Benacerraf 1965), as well as of propositions to unstructured or structured entities (see e.g. Bealer 1998 Melia 1992, King, Soames and Speaks 2014). This paper sets out to solve the problem by canvassing what we may call the arbitrary reference strategy. The main claims of such strategy are two. First: we do not know which objects are the referents of proposition and numerical terms since their reference is fixed arbitrarily. Secondly: our ignorance of which object is picked out as the referent does not entail that no object is referred to by the relevant expression. Different articulations of the strategy are assessed, and a new one is defended.
\end{abstract}

\section{The problem of arbitrary identification for reductionist realisms}

Some of the reductionist realist accounts of certain entities advanced in contemporary analytic philosophy seem to be affected by structurally similar problems, chief among them the problem of arbitrary identification. The problem gets off the ground when you:

(i) Specify the reductive framework within which the reduction should take place.

(ii) Ensure multiplicity, viz. make sure that there exists more than one candidate 
reduction.

(iii) Ensure equal adequacy, viz. make sure that all candidate reductions are equally adequate.

In his 1965 seminal article 'What numbers could not be', Paul Benacerraf shows how conditions (i)-(iii) can be satisfied by focusing on set-theoretic reductionist accounts of the natural numbers.

Let us begin with taking set theory to be the target reductive framework. Benacerraf claims that in order for any account of the natural numbers (no matter whether reductionist or non-reductionist in kind) to be adequate, two requirements must be met:

- The account should provide a model of the Dedekind-Peano axioms, thereby entailing the truth of the standard theorems of arithmetic.

- The account should explain cardinality in terms of counting.

These two requirements constitute what I shall call the arithmetic adequacy constraint. Benacerraf shows that there exists more than one arithmetically adequate set-theoretic reduction by focusing on two ways of stating the recursive clause ordering the settheoretic progression due to von Neumann and Zermelo. According to von Neumann's clause, the successor of a set $s$ is the union $s \cup\{s\}$. By contrast, according to Zermelo's, the successor of a set $s$ is the singleton $\{s\}$. Since both progressions satisfy the arithmetic adequacy constraint, there are multiple and equally set-theoretical reductions of the natural numbers to sets. Thus, Benacerraf contends that there is no non-arbitrary reason that enables us to establish which $\omega$-sequence is the natural numbers. This is, in kernel form, the problem of arbitrary identification for set-theoretic reductionist realism about arithmetic.

In this paper, I set out to solve the problem of arbitrary identification by canvassing what I shall call the arbitrary reference strategy. The main claims of such strategy are two. First: we do not know which objects are the referents of proposition and numerical terms since their reference is fixed arbitrarily. Secondly: our ignorance of which object is picked out as the referent does not entail that no object is referred to by the relevant 
expression.

Before articulating the arbitrary reference strategy, a few preliminaries are in order. First, I shall explain how the same problem can be raised against another central reductionist enterprise in analytic philosophy: that of explaining what propositions are. Secondly, I shall motivate the project of solving the problem of arbitrary identification on behalf of such reductionist views.

Several authors (e.g. Bealer 1998, Jubien 2001, King 2007, King, Soames and Speaks 2014, Melia 1992) have followed the lead of Benacerraf and raised the problem of arbitrary identification against reductionist views of propositions. The problem has been traditionally raised against views taking propositions to be unstructured abstract entities or structured entities conceived of in a Russellian or in a Fregean way. By way of illustration, let us focus on the best-known unstructured account which takes propositions to be world-theoretic abstract objects. ${ }^{1}$

Let us take possible worlds semantics to be the target reductive framework. Within this framework, we might say that a proposition is a function from possible worlds to truthvalues:

$$
\lambda w . \llbracket \varphi \rrbracket^{w}: W \rightarrow\{0,1\}
$$

However, at this function corresponds exactly one set, i.e. the set of those elements the function assigns 1 . So, a proposition might also be the set of possible worlds at which the sentence in question is true:

$$
\left\{w \mid \llbracket \varphi \rrbracket^{w}=1\right\}
$$

We therefore have the multiplicity ingredient: we can reduce propositions to more than one world-theoretic entity.

The last ingredient to add is what I have called 'equal adequacy'. Let me notice that all the extant discussions of the problem of arbitrary identification for propositions overlook the question of how equal adequacy is ensured. The typical move in the debate is to mention the isomorphism between sets and characteristic functions and conclude that we cannot choose one over the other in a non-arbitrary way. Yet, this is not sufficient to explain why both kinds of entities are equally adequate propositions-candidates. Therefore, I deem useful to show how to properly raise the problem of arbitrary 
identification for propositions.

I shall proceed first, by singling out what I shall call the proposition-properties, viz. the properties a given entity should have in order to be a proposition. ${ }^{2}$ Secondly, I argue that the adequacy constraint arising out of these proposition-properties can be, at least in principle, met by the candidate reductions.

Proponents of different brands of propositional realism such as Bealer (1998), King, Soames, and Speaks (2014), and Stalnaker (1999), all agree that in order for an entity to be a proposition, the entity must be:

(1) The compositional semantic value of sentences;

(2) the object of propositional attitudes;

(3) the content of illocutionary acts;

(4) the bearer of truth and modal properties;

(5) what we ascribe to others in attitude reports;

The conjunction of these statements provides what I shall call the propositional adequacy constraint that any account of propositions (no matter whether reductionist or not) must meet in order to be adequate.

At this stage, however, one might wonder whether the supporter of world-theoretic unstructured accounts of propositions can solve the problem of arbitrary identification by alleging that, since it is far from obvious that a single worldly entity can really satisfy the propositional adequacy constraint, such constraint is prejudicial against world-theoretic accounts of propositions. To elaborate on this train of thought, it is useful to pause on David Lewis's discussion of some of the proposition-properties mentioned above (see e.g. Lewis 1979, 1980).

As is well-known, Lewis maintains that while only modally neutral contents (viz. sets of possible worlds or their characteristic functions) can be the content of illocutionary acts, he maintains that we need finer-grained contents both to account for the presence in natural language of certain temporal and location expressions which behave as intensional operators, ${ }^{3}$ and to make sense of the special nature of first-person (de se) attitudes. Now, it seems safe to contend that, in order to have a distinctive challenge of 
arbitrary identification, the challenge has to trade upon the existence of multiple and equally adequate reductions, rather than on the fact that no adequate reduction can in the end be provided. So, since Lewis explicitly denies that there can be a single worldly entity playing all the proposition-roles, a Lewisean supporter of world-theoretic accounts of propositions could take the propositional adequacy constraint to be prejudicial against her view.

However, closer inspection reveals that this move does not enable the supporter of worldtheoretic accounts to avoid the problem of arbitrary identification. To see why, let us focus on the following proposition-properties of being:

(3) The content of illocutionary acts;

(4) the bearer of truth and modal properties;

(5) what we ascribe to others in attitude reports.

A supporter of world-theoretic unstructured accounts influenced by Lewis's considerations maintains that only modally neutral contents can play these roles. Yet, since sets of possible worlds and functions from possible worlds to truth-values are equally good candidate reductions for modally neutral contents, and since they are different entities, the problem of arbitrary identification kicks in again.

The same point holds if we focus on a different combination, e.g. that involving the first, the fourth, and the fifth proposition-properties. According to Lewis, only modally and temporally neutral contents can have these properties. Yet, such contents can be reduced to both sets of time-world pairs and functions from world-time pairs to truth-values. This teaches us a more general lesson: unstructured accounts of propositions are vulnerable to the problem of arbitrary identification no matter which version of the propositional adequacy constraint they adopt.

As has emerged from the foregoing discussion, the problem of arbitrary identification targets specific adequacy-based, as it were, reductionist realist accounts of certain entities, such as numbers and propositions. ${ }^{4}$ So, the problem vanishes if we endorse nonreductionist realist accounts of these entities; or if we pursue a different kind of reductionist project which doesn't rest solely on adequacy considerations; ${ }^{5}$ or else, if we 
give up the claim that these entities exist.

The aim of this paper, however, is to offer a solution to the problem of arbitrary identification on behalf of adequacy-based reductionist realism. One might therefore wonder: why save this brand of reductionism at all?

First, reductionist realisms are ontologically parsimonious: by reducing propositions and numbers to entities that are already part of our ontological inventory, such as sets, functions, ordered pairs, and the like, we are not forced to unduly inflate our ontology.

Secondly, accounting for propositions and numbers in the adequacy-based way ensures explanatory innocence. Numbers and propositions play certain roles, and there is little if any - disagreement amongst philosophers of arithmetic and philosophers of language about the fact that they play the roles described by the arithmetic and the propositional adequacy constraints. So, if we managed to tell what numbers and propositions are solely on the basis of something everybody agrees on, our explanation of the nature of such entities would exhibit significant explanatory innocence.

Thirdly, reductionist accounts of this sort might have further specific theoretical benefits. To illustrate this point briefly, consider a version of the hard problem of the unity of proposition asking us to explain why propositions have truth-conditions and are truth-apt (call this the representation problem $)^{6}$. Speaks (2014), and Stalnaker (1976), (1999), who defend reductive accounts of propositions which are affected by the problem of arbitrary identification, offer an elegant deflationary explanation of the representation problem. ${ }^{7}$ Stalnaker maintains that propositions are world-theoretic entities which are given by ways or properties the world might have (see Stalnaker 1976), whereas Speaks (2014) defends the view that propositions are identical to such properties. On Speaks's and Stalnaker's views, explaining why the proposition that Desdemona loves Cassio is true or false depending on whether she loves him or not amounts to explaining why the property of loving Cassio applies to Desdemona. The explanation is easy: properties, by their nature, apply to things. Punkt. Nothing more needs to be said about this. Hence, the representation problem can be successfully solved within Speaks's and Stalnaker's reductionist frameworks. Thus, since rescuing (at least some) adequacy-based reductionist views of propositions from the problem of arbitrary identification might come with further significant theoretical payoffs, we have an additional reason to take up 
the problem and solve it. ${ }^{8}$

Having clarified how to raise the problem of arbitrary identification and why we have reasons to solve it, we can turn now to discuss its solution.

\section{Solving the problem: the arbitrary reference strategy}

The remainder of this paper offers a full articulation of a specific strategy to solve the problem of arbitrary identification, namely the arbitrary reference strategy. Here is a concise statement of it. ${ }^{9}$ The reference of a numerical or a proposition term arbitrarily picks out a single determinate object of the domain of candidate reductions fixed by a given reductive framework (e.g. sets for numbers, world-theoretic entities for propositions, and so on). The fact that the reference is fixed arbitrarily is what explains why we do not know the referents of such expressions. Accordingly, the problem of arbitrary identification is solved as follows: the supporter of the problem of arbitrary identification is right in holding that we do not possess any reason to prefer one candidate reduction over the other as the correct one, for we do not know which objects numbersand propositions-naming expressions refer to. However, from our ignorance of the expressions' referents it does not follow that such expressions do not refer. That is to say, our ignorance of which candidate reduction is the correct one does not entail that there is no correct reduction at all.

A first clear statement of the arbitrary reference strategy can be found in Breckenridge and Magidor (2012: 397), even though they devote little space to the problem of arbitrary identification. Before them, John P. Burgess, in a no longer available 2009 manuscript

titled 'Putting structuralism in its place', touches upon the arbitrary reference strategy in the context of problems of arbitrary identification arising in various branches of mathematics.

Despite the increasing interest in arbitrary reference, though, I suspect that the strategy just sketched does not enjoy much pre-theoretical support. The main reason, I conjecture, is that the usual examples of referential expressions are such that we can somehow be in touch, either via direct acquaintance relations or through causal chains of communication 
of the sort envisaged by Kripke, with the objects referred to by such expressions. By contrast, the arbitrary reference strategy holds that the reference of such expressions is fixed, but we do not know how and we do not know which objects get to be the referents of such expressions. 'That's a kind of magic', a critic might urge.

To counter the initial scepticism and evaluate the prospects of the arbitrary reference strategy, I submit that two main questions should be addressed.

First: 'Since the thesis of arbitrary reference maintains that numbers- and propositionsnaming expressions pick out single determinate objects, it seems legitimate to ask: in virtue of what numbers- and propositions-naming expressions refer to a single determinate object?'. Call this the metasemantic question. Metasemantic questions are questions such as: 'In virtue of what a given expression receive the semantics it receives?'; 'What determines the semantic fact that a given expression has a such-andsuch meaning?'. Accordingly, the metasemantic question about propositions-naming expressions can be raised as follows: 'What determines which of the various worldtheoretic entities is picked out as the referent of a proposition-naming expression?'.

The second question can be formulated as follows: 'Why does arbitrary reference explain our ignorance of the referents of numerical and proposition terms?'. Call this the epistemic question.

It is plausible to think that different answers to the metasemantic question might give rise to different answers to the epistemic question. So, since answering the latter at least partly depends on answering the former, it makes sense to take the metasemantic question first.

As far as I can see, two answers to the metasemantic question can be found in the literature on arbitrary reference. I shall now present them and raise some concerns which motivate the project of looking for an alternative way of understanding the arbitrary reference strategy.

First answer: Breckenridge and Magidor (2012) handle the metasemantic question by endorsing what I shall call semantic primitivism, namely the view that it is a primitive (or fundamental) semantic fact that an arbitrarily referring expression picks out a given object. More specifically, semantic primitivism rejects the widely held view that semantic facts supervene upon use facts, viz. facts such as the speakers' dispositions to use the 
expression in a given scenario, the physical structure of the environment, and so on. As a consequence, the epistemic question is answered as follows. If semantic primitivism about arbitrary reference is correct, and we also assume that we cannot have knowledge of semantic facts unless we have knowledge of the use facts upon which they supervene, from the fact that numerical and proposition terms refer arbitrarily it follows that we cannot know the semantic fact that these terms refer to such and such objects.

I will come back to semantic primitivism below. For the time being, however, let me just flag that, despite the recent attempt at establishing semantic primitivism made by Kearns and Magidor (2012), the denial of the supervenience thesis is, prima facie, very costly. In addition, one may retort that invoking primitive semantic facts is but an ad hoc move which does not solve the mystery of how the reference of numerical and proposition terms gets fixed. Thus, for the time being, I shall content myself with noticing that it would be unsurprising if one wanted to look for a way to develop the arbitrary reference strategy which does not subscribe to semantic primitivism. ${ }^{10,11}$

Second answer: Boccuni (2013), Carrara and Martino (2010) and Martino (2001) adopt what Martino (2001) has called Acts of Choice Semantics. ${ }^{12}$ In a nutshell, the Acts of Choice Semantics envisages a series of ideal agents, who are pictured as having direct access to the objects of the domain of discourse and who, through an arbitrary act of choice, are able to single out any object of the domain of discourse in an ostensive manner. Hence the reference of arbitrarily referring expressions is fixed via an ideal baptism. In the case of proposition and numerical terms, this is tantamount to saying that ideal agents are appropriately connected to the relevant abstract objects and choose which of these objects are the referents of the respective terms.

The Acts of Choice Semantics answers the metasemantic question by preserving the supervenience of semantic facts upon use facts, in that the semantic fact that an arbitrarily referring expression picks out a single determinate object rather than another is determined by the act of choice of an ideal agent. Moreover, it explains the arbitrariness of reference by appealing to an act of choice being performed. As a consequence, the epistemic question is answered as follows. If the Acts of Choice Semantics is correct, and we assume that we cannot have access to the choices of the ideal agents, from the fact that numerical and proposition terms refer arbitrarily it follows that we cannot know the 
semantic fact that these terms refer to such and such objects.

On reflection, however, even if the Acts of Choice Semantics has the advantage of being compatible with the supervenience thesis, it falls prey to a different worry. ${ }^{13}$ The Acts of Choice semantics is explicitly taken by its proponents to be an idealisation of the Kripkean idea of there to be an act of baptism whereby the reference of a proper name is fixed (see Carrara and Martino 2010: 305). Since the agents fixing the reference are mere idealisations, there is no actual domain of agents. This means that, as a matter of fact, no actual act of baptism is performed. But if no actual baptism is performed, and if the reference is only fixed ideally, as opposed to being fixed in reality, it seems that we should conclude that arbitrarily referring expressions do not, after all, refer. ${ }^{14}$

Let us take stock. The two answers to the metasemantic question just reviewed are contentious, though for different reasons. Since the answer to the epistemic question (at least partly) depends on the answer to the metasemantic question, it follows that even the epistemic question has not been satisfactorily addressed. However, since the solution to the problem of arbitrary identification offered by the arbitrary reference strategy stands or falls on the plausibility of the answers to the metasemantic and the epistemic question, one might conclude that the problem cannot be satisfactorily solved by this strategy. I think that this would be too hasty a conclusion, though. For we can answer the metasemantic and the epistemic question in a better way, or so I argue in the next section.

\section{The metasemantics of proposition and numerical terms}

I shall begin with an explanation of the reference-fixing mechanism of proposition and numerical terms, and then turn to answering the metasemantic question and the epistemic question.

\subsection{Explaining arbitrary reference-fixing}

The view I shall defend is that the reference of proposition and numerical terms is fixed via a definite description which picks out the object the relevant expression refers to. A nowadays famous example is that of the descriptive name 'Julius'. Gareth Evans coined 
this name by introducing it into the language via the description 'whoever invented the zip'. To generalise: a reference-fixing description tells us how an entity should look like in order for it to be a given expression's extension. Thus, in order to determine the reference-fixing descriptions of propositions- and numbers-naming expressions, a promising starting point is to look at the propositional adequacy constraint and the arithmetic adequacy constraint respectively, for such constraints pin down the properties an entity should have in order for it to be a proposition and number, respectively.

I therefore submit that the reference-fixing description of a proposition-naming expression ' $p$ ' is the following: 'the satisfier of the propositional adequacy constraint $s$ such that $s$ is true just in case $p$ '. Thus, if we take the proposition that Price is a goalkeeper, and we refer to it via the name 'PRICE', 'PRICE' picks out the satisfier of the propositional adequacy constraint $s$ such that $s$ is true just in case Price is a goalkeeper. Analogously, the reference-fixing description of a number-naming expression ' $n$ ' is this: 'the $n$th satisfier of the arithmetic adequacy constraint'. That is to say, the referencefixing description of numerical terms is established by looking at the place the object should occupy in a $\omega$-sequence in order to be the referent of a given numerical term (e.g. a numeral). So, ' 0 ' picks out the least element of the satisfier of the arithmetic adequacy constraint, ' 1 ' picks out the next to the least element of the satisfier of the arithmetic adequacy constraint, and so on and so forth.

To explain the workings of the reference-fixing mechanism just introduced, a careful analysis of the relevant definite descriptions is needed. Reference-fixing descriptions are usually analysed in the standard Russellian way. Yet, a moment of reflection shows that the Russellian analysis does not really clarify what it means for an expression to have its reference fixed arbitrarily. As is well-known, Russell conceived of definite descriptions as incomplete symbols that have meaning only if used in a sentence. So, a sentence such as 'The King of France is bald' expresses the proposition that there exists a unique object that is the king of France and that object is bald. Yet, given the presence of multiple and equally adequate candidate referents for proposition and numerical terms, the Russellian analysis does not say anything about how, via the relevant description, we manage to pick out just one object amongst such candidates as the referent of the target term.

To make progress with this issue, I propose embracing an alternative analysis of definite 
descriptions, the so-called Hilbertian analysis. ${ }^{15}$ The Hilbertian analysis formalises a definite description such as 'The $F$ ' by using Hilbert's $\varepsilon$-term this way: $\varepsilon x F x$. Syntactically, the $\varepsilon$-term is a variable-binding operator that, just like Russell's $l$-term, allows us to form a term from a sentential form. That is to say, if $F$ is a formula and $x$ a variable, $\varepsilon x F$ is a term.

Let an epsilon calculus be any deductive system describing the logical behavior of the $\varepsilon$ term. An epsilon calculus consists of the usual axioms and deduction rules of first-order logic plus the following two axioms:

$$
\begin{aligned}
& \text { (A1) } F(t) \rightarrow F(\varepsilon x F(x)) \\
& \text { (A2) } \forall x(F(x) \leftrightarrow G(x)) \rightarrow \varepsilon_{X} F(x)=\varepsilon_{\mathrm{X}} G(x)
\end{aligned}
$$

The first axiom scheme introduces the $\varepsilon$-term into the language. The second axiom, sometimes called the extensionality axiom, says that if two formulas are equivalent then their $\varepsilon$-expressions are identical.

Semantically, the $\varepsilon$-term behaves as a choice function. A choice function on a collection $F$ of nonempty sets assigns to each set $s$ in $F$ one element of $s$. If there are any $F s$, then $\varepsilon x F x$ selects one object out of the $F s$. By contrast, if there are not any, then $\varepsilon x F x$ selects one object from the whole universe.

In order to provide an analysis of definite (as opposed to indefinite) descriptions by using Hilbert's $\varepsilon$-term, two presuppositions must be in place (see Slater 1988). First, there are $F s$; secondly, there is a unique object that is $F$. If we presuppose both things, the description 'The $F$ ' means, roughly, something like 'the unique chosen object $x$ such that $x$ is $F^{3}{ }^{16}$

Having clarified this, let us now deploy the Hilbertian reading to analyse the definite description 'the satisfier of the propositional adequacy constraint', which is the stable part of all reference-fixing descriptions of proposition terms. The reductionist realist projects we are considering do presuppose the existence of propositions. It also presupposes that ' $p$ ' picks out a unique object. The arbitrary reference strategy is precisely meant to explain how to redeem such a presupposition while, at the same time, granting that there be multiple and equally adequate candidate entities ' $p$ ' could refer to. 
That being said, let us take now the collection of all objects satisfying the propositional adequacy constraint. The choice function selects one object out of the collection of all objects satisfying the propositional adequacy constraint completely randomly. Hence, the reference-fixing description of a proposition-naming expression ' $p$ ' selects a worldly entity satisfying the constraint as the referent of the expression such that the selected worldly entity is true just in case $p$. So, a proposition-naming expression such as ' $p$ ' is a device of direct reference, for it contributes an object to the truth-conditions of the sentence in which it occurs. However, we do not know whether it is a set of possible worlds that obtain just in case $p$, or whether it is the characteristic function of such set.

Let us turn now to numerical terms. The description 'the satisfier of the arithmetic adequacy constraint', which is the stable part of all reference-fixing descriptions of numerical terms, picks out a sequence out of the collection of all sequences satisfying the arithmetic adequacy constraint. Let us take, for instance, the description 'The next to the least element of the satisfier of the arithmetic adequacy constraint', which is the reference-fixing description of the numeral ' 1 ': ' 1 ' refers to the next to the least element of the chosen set-theoretic progression that satisfies the arithmetic adequacy constraint. However, we do not know whether the randomly selected sequence is Zermelo's, von Neumann's, or any other sequence.

I have unpacked the idea that the reference of proposition and numerical terms is fixed in an arbitrary way by appealing to Hilbert's $\varepsilon$-term. I hasten to acknowledge that the connections between Hilbert's $\varepsilon$-term and the idea of arbitrariness have already been pointed out by various authors. Slater (1988) mentions the possibility of analysing arbitrary parameters in instantial reasoning via Hilbert's $\varepsilon$-term, but he never addresses the problem of arbitrary identification. Burgess (2009) mentions only very briefly the possibility of harnessing Hilbert's $\varepsilon$-term to solve the problem of arbitrary identification. So, I regard the previous proposal as taking the inspiration from Slater's and Burgess' insights.

$\mathrm{Be}$ that as it may, it appears fair to say that the prospects of the arbitrary reference strategy chiefly depend on answering the metasemantic question which, in turn, enables us to answer the epistemic question. So, a proper treatment of the metasemantic question is called for. To this task I shall turn in the next section. 


\subsection{Answering the metasemantic question and the epistemic question}

The view I have defended in the previous section has it that numerical and proposition terms receive their reference in virtue of a choice function that selects their referents out of the collection of all the candidate referents. This offers a preliminary answer to the metasemantic question. On reflection, though, the choice function fixing the reference of propositions- and numbers-naming expressions is an arbitrary one. That is to say, it could well be the case that a numeral ' $n$ ' is associated with the reference-fixer ' $c x F x$ ' picking out a Zermelo set, whereas a numeral ' $m$ ' is associated with the reference-fixer ' $\varepsilon x G x$ ' picking out a von Neumann set. So, what guarantees that that the choice function selecting the invariant part of all reference-fixing descriptions is the same across all such descriptions? This sends back to the problem of choosing an arbitrary choice function. This suggests that, given the explanation of arbitrary reference-fixing I offer, the metasemantic question should be reformulated as follows: "What determines the choice function that determines the reference of the description 'the satisfier of the propositional adequacy constraint'?". ${ }^{17}$

This question can be answered by delving a little more into metasemantic considerations. A somewhat successful metasemantic approach has it that semantic facts supervene upon use facts and naturalness facts. This view has been famously put forward by David Lewis in his seminal paper 'New work for a theory of universals', and subsequently defended and developed by, amongst others, Sider (2001, 2011) and Weatherson (2003). Lewis's idea, in a nutshell, is that there are different levels of reality ordered on the basis of their degree of naturalness. To illustrate the idea, let us focus on properties. At the very bottom there are what Lewis calls perfectly natural properties, viz. the properties that carve out the joints of nature. Lewis takes the notion of naturalness to be primitive, but a commonly accepted heuristic to compare different degrees of naturalness consists in focusing on the degree of objective similarity exhibited by the members of a given set (Bear in mind that, for Lewis, properties are sets). To use a nowadays famous example: the property of being green is more natural than the property of being grue because, even though green things form a rather heterogenous set, they are more alike than the grue 
things are. ${ }^{18}$

The Lewisean metasemantic view has it that the referent of a term is the most natural entity that is more-or-less consistent with the usage of the term. Take the predicate 'green': while both green and grue fit our dispositions to use 'green', since green is a more natural property than grue is, green is a more eligible candidate referent for 'green' than grue is. This approach is extended by Lewis himself to the reference of singular terms, as is witnessed by the example of the cat referred to by 'Bruce' in Lewis (1983). ${ }^{19}$ By adopting a Lewisean metasemantics, we can maintain that the semantic fact to the effect that a proposition-naming expression ' $p$ ' refers to the chosen object $s$ satisfying the propositional adequacy constraint such that $s$ is true just in case p supervenes upon use facts and naturalness facts.

I propose to regard use facts as those facts in virtue of which the propositional adequacy constraint obtains. So, roughly put, the use facts pertaining to propositions-naming expressions are constituted by the way a group of experts in our community, i.e. semanticists and philosophers of language, use such expressions. Since we have seen that philosophers of language and semanticists use proposition terms to talk about those entities having the properties that constitute the propositional adequacy constraint, it seems safe to claim that the relevant use facts are encapsulated in the propositional adequacy constraint.

Use facts enable us to rule out clear cases of entities which cannot act as candidate referents for propositions-naming expressions. For instance, given the way philosophers and semanticists use propositions-naming expressions, syntactic entities such as sentences can be ruled out as potential candidate referents for proposition terms. Moreover, since the propositional adequacy constraint is the stable part of the referencefixing descriptions of proposition terms, it is clear that use facts play a role in determining the meaning of such terms.

Given the analysis of the relevant definite descriptions offered above, the use of proposition terms does not require us to choose one specific world-theoretic object rather than another. That is to say, since both sets of possible worlds and their characteristic functions are able to do the job, referents of propositions-naming expressions can be chosen completely randomly. To repeat, what really matters is that the chosen entity 
possesses the relevant proposition-properties constituting the propositional adequacy constraint. $^{20}$

The foregoing explains what role use facts play in determining the meaning of proposition terms. Use facts determine which definite descriptions fix the reference of such terms. Clearly, however, facts about the usage of propositions-naming expressions are not so fine-grained as to determine which choice function does the work of fixing the referent, for there might be different choice functions which would fit such an usage. Here the second component of the Lewisean metasemantic approach comes into play. If entities are ordered on the basis of their naturalness, and if semantic facts supervene upon both use facts and naturalness facts, the supporter of the arbitrary reference strategy can maintain that there is a choice function that is more natural than any other one, even though we do not know which one it is. Of course, this requires an extension of the naturalness talk to abstracta, i.e. choice functions. Surely, while we can have a grasp of the differences in naturalness between entities populating the concrete reality in virtue of our access to such entities and observation of their objective similarities, we cannot have a similar grasp of the differences in naturalness of abstract objects, in that we lack a plausible epistemology of abstracta. This, I contend, is what happens with the choice function contributing to fixing the reference of proposition and number terms. The claim I am making is that there is a privileged choice function such that the satisfier of the arithmetic adequacy constraint is the same for all numerals Yet, since we do not have an epistemic access to the domain of abstracta, we do not know which function it is.

To forestall misunderstandings: I am not claiming here that there is a semantic fact $s f_{l}$, e.g. that ' $n$ ' refers to a Zermelo set, which is more natural than another semantic fact $s f_{2}$, e.g. that ' $n$ ' refers to a von Neumann set. Appealing to naturalness at the level of semantic facts cannot solve the problem of arbitrary identification on behalf of the adequacy-based reductionist realist we are considering here. Let me explain.

It bears repeating that the reductionist realisms affected by the problem of arbitrary identification maintain the distinctive thesis that the only criteria to take into account while assessing whether a given entity is a number or a proposition are provided by the arithmetic and propositional adequacy constraints, respectively. So, if we said that, of the two options, functions from worlds to truth-values or sets of worlds, one of them is the 
more natural candidate entity for being a proposition but we just can't know which, we would be introducing another element, i.e. naturalness, which would help us decide which thing is a proposition and which thing is not. Yet, doing so amounts to violating the propositional reductionist credo that all there is to establishing the nature of propositions is given by the proposition-properties constituting the propositional adequacy constraint. Similarly, if we said that of the infinitely many set-theoretic progressions which satisfy the arithmetic adequacy constraint one is more natural than any other, we would be violating the arithmetic structuralist credo that the essence of numbers is given by their order-theoretic properties only.

However, the adequacy-based reductionist realist need not impose any constraint on how metasemantic facts should look like. So, the adequacy-based reductionist realist can clearly accept the claim I making to the effect there is a choice function - which, together with the use facts encapsulated in the relevant definite descriptions, determines the semantic fact that ' $n$ ' refers arbitrarily to the $n t h$ satisfier of the arithmetic adequacy constraint - that is more natural than any other. Thus, on the view I offer, naturalness is appealed to at the level of what fixes the non-semantic facts that are the supervenience base of semantic facts of the form: ' $n$ ' refers arbitrarily to the $n$th satisfier of the arithmetic adequacy constraint.

This completes my answer to the metasemantic question. In light of this, we can now address the epistemic question.

It must be kept in mind that the epistemic question asks why arbitrary reference explains our ignorance of the referents of proposition (and numerical) terms. My answer is as follows. The articulation of the arbitrary reference strategy on offer maintains that the reference of proposition terms is partly determined by naturalness facts. The specific naturalness facts appealed to are facts about choice functions. Since choice functions are abstract entities, and since we do not (yet) have a viable epistemology of abstract entities, we are currently unable to access the naturalness ordering of choice functions. So, since we are unable to know which choice function has contributed to fixing the referents of proposition (and numerical) terms. But if we are unable to know which choice function has picked out the objects, we cannot know which objects have been picked out as the referents of proposition (and numerical) terms. Hence, what explains our ignorance of the 
referents of proposition and numerical terms is our ignorance of the naturalness ordering of the functions which contribute to fixing the reference of such terms. ${ }^{21}$

\section{Assessing the (new) arbitrary reference strategy}

Now that the details of the new version of arbitrary reference strategy have been presented, we can turn to provide a costs-benefit analysis of the strategy. I shall first consider two questions about my version of the arbitrary reference strategy and then address two general concerns about the strategy itself.

\subsection{Two questions about my version of the strategy}

First question: One might wonder: 'Why is your view any better than Breckenridge and Magidor's and Martino's? More specifically, why should one accept your answer to the metasemantic question and, consequently, your answer to the epistemic question?'.

Answer: I diagnose the source of this concern in the suspicion that invoking naturalness facts about choice functions is as ad hoc as either taking semantic facts to be primitive or invoking ideal agents. I believe that this suspicion is misplaced. As a first general consideration, let me emphasise that the appeal to naturalness is independently motivated by Lewis's insightful approach to the nature of reality. Roughly put, if reference is a relation between an expression and an existing thing, then there is no ad-hocness in holding a view about why a given reference relation obtains which relies upon a given (and independently attractive) metaphysical picture. Secondly, it is worth keeping in

mind that naturalness facts play an explanatory role in addressing other major metasemantic problems, such as Kripkenstein's rule-following paradox, Putnam's modeltheoretic argument, Quine's indeterminacy of reference.

Having said that, let us compare the metasemantics I endorse with semantic primitivism. Kearns and Magidor (2012), who have put forward an extensive defence of semantic primitivism, stress that semantic primitivism has never been put on the table as a real option to solve the classical metasemantic problems mentioned in the previous paragraph. 
However, in a footnote (Kearns and Magidor 2012: fn. 3, p. 323) Kearns and Magidor explicitly claim that they 'assume' that a global form about scepticism about meaning is false (call this view semantic scepticism). So, their argument seems to run as follows: if global semantic scepticism is false, then there are semantic facts. If we reject the supervenience claim, then semantic facts are primitive (or fundamental). However, I cannot help but ask: how can we simply assume that semantic scepticism is false without begging the question against the Kripkensteinean sceptic?

It is indeed worth bearing in mind that Kripkenstein's rule-following paradox is meant to show that the supervenience of semantic facts upon use facts does not obtain. If we accept this conclusion, and if we do not want to appeal to naturalness facts, we have two options left: either we accept semantic scepticism, or we accept semantic primitivism. The question is: why should we accept the latter rather than the former? As far as I can see, beside the remark in the aforementioned footnote to the effect that they assume that global scepticism about meaning is false, Kearns and Magidor do not offer any answer to this question. And yet, this assumption simply begs the question against the Kripkensteinean sceptic. By contrast, the appeal to naturalness facts seems to be less an ad hoc response to the challenge raised by the semantic sceptic: such a response does not simply assume without argument that there are semantic facts, for it attempts at offering an explanation of why there are semantic facts which partly relies on an independently attractive metaphysical picture of reality. Thus the explanatory gain of locating the epistemological mystery, as it were, at the level of metasemantic facts, like my version of the arbitrary reference strategy does, as opposed to locating it at the level of semantic facts, as Breckenridge and Magidor's version of the strategy does, is that doing so enables us to avoid begging the question against the semantic sceptic.

Let us now briefly contrast the metasemantic picture upheld by the supporters of the Acts of Choice Semantics with the one I am recommending. The metasemantic view I defend is such that it preserves Martino's insight to the effect that the reference of arbitrarily referring expressions is chosen randomly. However, it does so without the costly commitment to there being ideal acts of baptism which do not fix the reference of such expressions in reality. On the version of the arbitrary reference strategy I have developed, the reference-fixing is not ideal, but real. 
Second question: One might worry that proposition and numerical terms have not been introduced into the language via the definite descriptions I take to be playing the role of fixing their reference. One might indeed worry that once the numeral ' 1 ' has been introduced in the Hindu-Arabic tradition, the relevant description 'The $F$ ' was certainly different from the one proposed here. ${ }^{22}$ How to make sense of this?

Answer: To begin with, let me draw our attention to the fact that numerals and other numbers-naming expressions can have various uses. My aim here is solely to capture the use that is made in the formal theory we call arithmetic, that is, the theory of the natural numbers as defined by the Dedekind-Peano axioms. I will qualify this claim below, while responding to a worry about the general shape of the arbitrary reference strategy. For the time being, however, let me emphasise that the boundaries of arithmetic discourse have been fixed only in the 19th century thanks to the work of (among others) Dedekind and Peano, who came up with a formally precise definition of the numbers. So, past uses of the numerals are not our concern here.

Having said that, I also want to suggest that the reference-fixing descriptions deployed by my account do bear some relation to uses of numerals made before the appearance of the Dedekind-Peano axioms. It seems indeed plausible to maintain that numerals were originally introduced to refer to something we count with, as it were. On closer inspection, this rough description may be seen as the ancestor of the idea that numbers are those entities that are used to count collections. As has emerged previously, this is one of the two clauses of the arithmetic adequacy constraint.

This suggests that the process of reference-fixing in the case of numerals originated many centuries ago, and gradually unfolded until the formally precise formulation of the Dedekind-Peano axioms and a proper understanding the concept of cardinality were available. Thus, even if a numeral ' $n$ ' was not introduced in the language via the definite description 'the $n$th satisfier of the arithmetic adequacy constraint', such a description is the outcome of a gradual process of reference-fixing which has its roots in the first historical introduction of numerals and its complete fulfillment in the formulation of the arithmetic adequacy constraint.

The foregoing train of thought is further supported by the observation that referencefixing need not be a matter of quick and easy stipulation. Surely, there are cases in which 
the process of reference-fixing is a farily easy stipulative act, e.g. Evans's case of 'Julius'. In other cases, the process could take a couple of years and involve a small community. Take the case of 'Jack the Ripper'. The name has been introduced in 1888 via a certain definite description, say 'the murderer of prostitutes in the East End of London'. Then, being more specific about the location modified the description in, say, this way: 'the murderer of five prostitutes in the Whitechapel district'. Around 1891, 'Jake the Ripper' referred to whoever satisfied the description 'the murderer of eleven prostitutes in the Whitechapel district between April 1888 and February 1891'. Thus, the process of reference-fixing of 'Jake the Ripper' was gradual and the relevant referencefixing description changed depending on the new information acquired by London's police during the investigation. In the case of numerals, the process lasted several centuries and involved different communities of mathematicians.

Let us turn now to two more general questions about the arbitrary reference strategy.

\subsection{Two questions about the strategy in general}

Third question: The arbitrary reference strategy assumes that both propositions- and numbers- naming expressions are referential. On the basis of this assumption, it provides an account of how they refer that enables the reductionist to respond to the problem of arbitrary identification. And yet, it is doubtful that proposition and numerical terms always behave as referential expressions in a number of different natural language constructions. ${ }^{23}$ How does a supporter of the arbitrary reference strategy defend the referentiality assumption?

Answer: As said above, I acknowledge the existence of a variety of uses of proposition and numerical terms, some of which might well not be referential. Yet, I believe that this does not undermine the arbitrary reference strategy. To see why, we should bear in mind that the strategy is put to service to the adequacy-based reductionist realist. Thus, in order to pursue a proper assessment of the strategy, it is important to understand the kind of project the adequacy-based reductionist realist is after.

As far as I can tell, the reductionist is no natural language linguist: her aim is not to account for all uses of numbers- or propositions-naming expressions. Rather, her focus is 
on those uses which are integral to the account of the nature of the target discourse, e.g. arithmetic discourse and semantic discourse. Thus, we can set aside many natural language constructions and focus instead on those constructions used by working mathematicians and semanticists. To illustrate what I have in mind, take the case of arithmetic discourse. It is a well-known result that every arithmetic sentence can be paraphrased by sentences containing first-order quantifiers, the identity symbol, the setmembership relation, and the expressions ' $N$ ', ' 0 ', ' 1 ', '+', ' $\times$ '. The arithmetic realist contends that ' $N$ ', ' 0 ', ' 1 ' behave as proper names: ' 0 ' and ' 1 ' refer to objects which are elements of ' $N$ ', while ' + ' and ' $\times$ ' denote functions. The realist who is also a settheoretic reductionist maintains that ' $N$ ' refers to a set of sets of which the referents of ' 0 ' and ' 1 ' are elements. Since we could state the core reductionist thesis in this minimal way, what matters is to deploy the arbitrary reference strategy only with regard to the referential uses of these expressions. Thus, the existence of different (and non-referential) uses of numbers-naming expressions in different natural languages does not undermine the deployment of the arbitrary reference strategy.

As for semantic discourse, things are more complex. To begin with, we should acknowledge that the propositional realist uses three types of expressions to refer to propositions:

(a) Proper names: $\mathrm{p}$

(b) Definite descriptions: the proposition $p$

(c) 'That'-clauses: that $p$

As is well-known, the question whether all uses of proper names, definite descriptions, and 'that'-clauses are referential is hotly debated. To illustrate the difficulty, take proper names. In light of the recent debate between referentialism and predicativism, ${ }^{24}$ one might argue that there are uses of proper names which are apparently predicative as opposed to referential, and the best theory that accounts for all these uses is predicativism.

However, we can sidestep such predicative uses by contending that the propositional reductionist, just like the arithmetic reductionist, tries to capture the use of proposition 
names within semantic theory. Since those uses are referential, the propositional reductionist can focus on them only. The same sort of consideration can be deployed in the case of definite descriptions, for it is widely acknowledged that they have both referential and attributive uses.

We are therefore left with 'that'-clauses. Surely, semanticists disagree over whether 'that'-clauses have referential uses at all, so the propositional reductionist cannot simply maintain that such expressions, when used in semantic theorising, have a referential use only. Thus, let us concede, for the sake of argument, that 'that'-clauses are not referential expressions. How to analyse them?

A prominent non-referential analysis is the one offered in Recanati (2004). Recanati proposes to treat 'that'-clauses as restricted existential quantifiers and paraphrase a 'that'clause such as 'that $p$ ' as follows: 'For some $p$ such that $\mathrm{p}$ is true iff $S$ ', where ' $p$ ' is an objectual variable ranging over propositions and ' $S$ ' stands for the sentence embedded in the 'that'-clause.

Interestingly, a version of the problem of arbitrary identification can be raised even if we accept Recanati's quantificational analysis of 'that'-clauses. One might indeed wonder: do the objectual variables range over sets of possible worlds, or their characteristic functions? Insofar as there is no non-arbitrary reason to prefer one domain of discourse over the other, we might conclude that the domain of discourse for the quantifier is empty.

Fortunately, however, I believe that the specific version of the arbitrary reference strategy I have been developing can be suitably modified so as to block this alternative version of the problem of arbitrary identification. One of the noteworthy features of epsilon calculi is that, given the two distinctive axioms of such calculi mentioned above, we can explicitly define quantifiers as follows:

$$
\begin{aligned}
& (\exists) \exists x F x \leftrightarrow F \varepsilon x F x \\
& (\forall) \forall x F x \leftrightarrow F \varepsilon x \neg F x
\end{aligned}
$$

We can therefore take the sentence 'For some $p$ such that $\mathrm{p}$ is true iff $S$ ' to mean the following: 'For the chosen object $p$ satisfying the propositional adequacy constraint such 
that $p$ is true iff $S$ ', where ' $p$ ' is an objectual variable ranging over only one kind of world-theoretic entity (but we do not know which one it is). This enables us to randomly restrict the range of quantification of the objectual variables involved in the analysis of the meaning of 'that'-clauses, thereby mirroring what we did with the reference-fixing mechanism of referential proposition terms.

The foregoing shows that appealing to a choice-theoretic semantics can solve the problem of arbitrary identification even if we strip off its referentialist component. This ensures a certain theoretical flexibility in the deployment of the general strategy here outlined that cannot but be welcomed by the propositional reductionist.

Fourth question: Finally, one might wonder: 'Are there any other strategies for solving the problem of arbitrary identification on behalf of reductionist realists? If so, what's the advantage of the arbitrary reference strategy over these alternatives?'.

Answer: There are other solutions on the market, but they have already received some criticism. In a 1999 paper, Joseph G. Moore considers and rejects a strategy relying on the idea that the reference of proposition and numerical terms is indeterminate. The notion of indeterminacy can be cashed out in both semantic and ontic terms. As for the semantic interpretation, the driving thought is that the correct semantics of, say, propositions-naming expressions is supervaluationist, and the multiple candidate reductions act as different but equally legitimate way of sharpening the meaning of a proposition term such as ' $p$ '. Moore, however, argues that various candidate propositions (sets of possible worlds, functions from worlds to truth-values, sets of ordered pairs, and so on) cannot serve as sharpenings of the meaning of propositions terms (Moore 1999: 244-246). By contrast, the ontic interpretation of indeterminacy gives rise to the idea that proposition terms refer to vague objects. Besides mentioning some general worries about vague objects, Moore lays out a modal argument which rules out the possibility of regarding propositions as vague objects (Moore 1999: 247-248).

A careful assessment of Moore's arguments would lead us astray. However, let me just flag that since the arbitrary reference strategy nowhere appeals to (ontic or semantic) indeterminacy, it is immune to the worries raised by Moore regarding vague objects and candidate sharpenings. Thus, it seems that the arbitrary reference strategy enables us to make significant progress with respect to the evaluation of reductionist accounts: insofar 
as one wants to undermine such accounts by raising the problem of arbitrary identification, one has to take issue with the new version of arbitrary reference strategy and show where it fails.

\section{Conclusion}

The paper has offered a solution to the problem of arbitrary identification by articulating the semantic and metasemantic details of the arbitrary reference strategy. Proposition and numerical terms pick out single determinate objects arbitrarily. The reference-fixing mechanism of such terms involves complex definite descriptions. The reference of a numerical term such ' 1 ' is this: 'The next to the least element of the satisfier of the arithmetic adequacy constraint'. The reference-fixing description is complex since it presents a changing component and a stable component. The changing component, which is expressed here by 'The next to the least element', changes depending on which numerical terms we focus on. The stable component, which is expressed here by the embedded definite description 'The satisfier of the arithmetic adequacy constraint' is formalised via Hilbert's $\varepsilon$-term, whose interpretation is a choice function. Thus, if we take set theory to be the target reductive framework of arithmetic, the choice function selects randomly one amongst the (infinitely many) set-theoretic progressions which satisfy the arithmetic adequacy constraint. The same holds, mutatis mutandis, for proposition terms.

At the metasemantic level, it is contended that what fixes the reference of such expressions are both use facts, i.e. facts about how working semanticists and mathematicians use proposition and numerical terms, and naturalness facts, i.e. facts about which choice function whereby part of the semantics of these terms gets fixed is the most natural one. Some possible objections to the arbitrary reference strategy have been addressed.

To conclude. I believe that adequacy-based reductionist realists about entities such as numbers and propositions should rejoice, for they have a novel and full-fledged response to the problem of arbitrary identification. To forestall misunderstandings, however, the discussion pursued in this paper is not meant to amount to a full vindication of (some of) 
these reductionist views. However, by showing how the problem of arbitrary identification can be successfully solved, I hope that this paper can be regarded as a first step towards such a vindication.

\section{Acknowledgements}

I would like to thank Francesca Boccuni, Samuele Chilovi, Berta Grimau, Daniel Morgan, Michael Murez, Hichem Naar, Marco Panza, François Recanati, and Andrea Sereni for their helpful comments on various parts of this paper. Special thanks go to Francesca for her advice and support at the early stages of this project. Versions of this paper have been presented at the Institut Jean Nicod's 2016 Mind and Language seminar, the $1^{\text {st }}$ FilMat Conference hosted by the University of San Raffaele-Milan, the XI SIFA Conference hosted by the University of l'Aquila, the XVIII TIF conference hosted by the University of Valencia. I would like those in attendance on these occasions for their feedback. I gratefully acknowledge the financial support of the Spanish Ministerio de Economia y Competitividad (MINECO) under grant agreement \#FFI2016-80588-R, the European Commission's Horizon 2020 programme under grant agreement H2020MSCA-ITN-2015-675415, the Juan de la Cierva and Beatriu de Pinós postdoctoral fellowship programmes (under grant agreements FCJI-2014-20227 and 2016BP00142), the CRÉ-GRIN postdoctoral fellowship programme.

\section{References}

Bealer, G. (1998). Propositions. Mind, 107(425), 1-32.

Benacerraf, P. (1965). What numbers could not be. The Philosophical Review, 74(1), 4773.

Boccuni, F. (2013). Plural logicism. Erkenntnis, 78(5), 1051-1067.

Breckenridge, W. \& Magidor, O. (2012). Arbitrary reference. Philosophical Studies, 158(3), 377-400. 
Cameron, R. P. (2010). Vagueness and naturalness. Erkenntnis, 72, 281-293.

Caplan, B. \& Tillman, C. (2013). Benacerraf's revenge. Philosophical Studies, 166, 119129.

Carrara, M. \& Martino, E. (2010). To be is to be the object of a possible act of choice. Studia Logica, 96, 289-313.

Clarke-Doane, J. (2008). Multiple reductions revisited. Philosophia Mathematica, 16, 244-255.

Dixon, S.T. \& Gilmore, C. (2016). Speaks's reduction of propositions to properties: a Benacerraf problem. Thought, 5(4), 275-284.

Dummett, M. (1973). Frege: Philosophy of Language. London, UK: Duckworth.

Eklund, M. (forthcoming). Regress, unity, facts, and propositions. Synthese, online first DOI: $10.1007 / \mathrm{s} 11229-016-1155-4$.

Fine, K. (1985). Reasoning with arbitrary objects. Oxford, UK: Blackwell.

Hilbert, D. \& Bernays, P. (1939). Grundlagen der Mathematik: Vol. II. Berlin-New York: Springer-Verlag.

Jeshion, R. (2015). Referentialism and predicativism about proper names. Erkenntnis, 80(2), 363-404.

Jubien, M. (2001). Propositions and the objects of thought. Philosophical Studies, 104, 47-62. 
Kearns, S. \& Magidor, O. (2012). Semantic sovereignty. Philosophy and Phenomenological Research, 85(2), 322-350.

King, J. C. (2007). The nature and structure of content. Oxford, UK: Oxford University Press.

---- (2014). Naturalized propositions. In J.C. King, S. Soames, \& J. Speaks, New Thinking About Propositions (pp. 47-70). Oxford, UK: Oxford University Press.

King, J.C., Soames, S. \& Speaks, J. (2014). New Thinking About Propositions. Oxford, UK: Oxford University Press.

Lewis, D. K. (1979). Attitudes de dicto and de se. In D. K. Lewis, Philosophical Papers: Vol. I (pp. 133-159). New York: Oxford University Press.

---- (1980). Index, context and content. In D. K. Lewis, Papers in Philosophical Logic (pp. 21-44). Cambridge, UK: Cambridge University Press.

---- (1983). New work for a theory of universals. Australasian Journal of Philosophy, 61(4), 343-377.

Martino, E. (2001). Arbitrary reference in mathematical reasoning. Topoi, 20, 65-77.

Melia, J. (1992). An alleged disanalogy between numbers and propositions. Analysis, 52(1), 46-48.

Moltmann, F. (2013). Abstract objects and the semantics of natural language. Oxford, UK: Oxford University Press.

Moore, J. G. (1999). Propositions, numbers, and the problem of arbitrary identification. Synthese, 120, 229-263. 
Ninan, D. (2010). Semantics and the objects of assertion. Linguistics and Philosophy, 33, 355-380.

Pettigrew, R. (2008). Platonism and aristotelianism in mathematics. Philosophia Mathematica, 16(3), 310-332.

Pickel, B. (forthcoming). Unity through truth. Synthese, online first DOI: 10.1007/s11229-016-1279-6.

Recanati, F. (2004). 'That'-clauses as existential quantifiers. Analysis, 64(3),: 229-235.

Sider, T. (2001). Personal identity and the limits of conceptual analysis. Philosophical Perspectives, 15, 189-209.

---- (2011). Writing the book of the world. Oxford, UK: Oxford University Press.

Slater, B. H. (1988). Hilbertian reference. Noûs, 22(2), 283-297.

Speaks, J. (2014). Propositions are properties of everything or nothing. In J.C. King, S. Soames, \& J. Speaks, New Thinking About Propositions (pp. 71-90). Oxford, UK: Oxford University Press.

Stalnaker, R. (1976). Possible worlds. Noûs, 10(1), 65-75.

---- (1999). Context and content. Oxford, UK: Oxford University Press.

Steinhart, E. (2002). Why numbers are sets. Synthese, 133(3), 343-361.

Weatherson, B. (2003). What good are counterexamples?. Philosophical Studies, 115, 131. 
1 Four quick points are in order. First, throughout the paper I will focus on the world-theoretic view of propositions, but my arguments should carry over to all the reductionist views about propositions affected by the problem of arbitrary identification, most notably the Russellian and Fregean structured accounts. Secondly, let me clarify that while Benacerraf took his argument to undermine arithmetic realism and provide an argument for a structuralist version of nominalism, there are some authors (e.g. Bealer and King) raising the problem against e.g. world-theoretic accounts of propositions while, at the same time, believing that the problem undermines these accounts without ipso facto undermining propositional realism. Thirdly, Caplan and Tillman (2013), and Dixon and Gilmore (2016) have argued, respectively, that King's $(2007,2014)$ and Speaks's (2014) new reductionist views are also affected by the problem of arbitrary identification. Fourthly, the problem of arbitrary identification affects also set-theoretic reductions of ordered pairs. These last two points indicate the pervasiveness of such a problem, and I indeed conjecture that the problem could emerge in other similar reductionist realist enterprises, even though I cannot argue for this point here.

2 I shall sometimes speak of proposition-roles instead of proposition-properties.

3 This is similar to Dummett's claim that we need to distinguish between assertoric content and ingredient sense (Dummett 1973). See Ninan (2010) for further discussion.

4 I will henceforth omit 'natural', for the paper focuses solely on the natural numbers.

5 For instance, Clarke-Doane (2008) and Steinhart (2002) have argued that there is more to establishing which settheoretic progression is the sequence of the numbers than adequacy considerations.

6 The label is Eklund's (Eklund forthcoming).

7 See also Pickel (forthcoming) for another deflationary explanation using structured propositions.

8 Of course, different reductionist views will claim to have more or different specific theoretical benefits. I won't go into a discussion of what such benefits may be.

9 The very idea of arbitrary reference has been primarily articulated to make sense of the semantic behaviour of arbitrary parameters in instantial reasoning. To illustrate this briefly, take existential instantiation: if we have derived $\exists x F(x)$ we can introduce a new parameter 'a' that does not occur in the general formula $\exists x F(x)$ or in any undischarged assumption supporting the formula, and assume $\mathrm{Fa}$. The complete generality of existential instantiation is achieved by letting the rule be valid whatever $a$ is. Boccuni (2013), Breckenridge and Magidor (2012), Carrara and Martino (2010), and Martino (2001) defend the contention that the parameter ' $a$ ' arbitrarily refers to an object of the domain of discourse satisfying $F(x)$, yet we do not know which object that is. Let me notice that this is not the only interpretation of the behaviour of arbitrary parameters in instantial reasoning (see e.g. Fine 1985 and Pettigrew 2008 for other interpretations). However, it is not my aim here to assess different views about arbitrary parameters in instantial reasoning.

10 Given semantic primitivism, and given that propositions are semanticalia, as it were, it seems that Breckenridge and Magidor should be nonreductionist about propositions. Thanks to Samuele Chilovi and Michael Murez for discussion of this point.

11 I won't focus here on the second premise of the semantic primitivist answer to the epistemic question sketched above to the effect that we cannot know semantic facts unless we have knowledge of the use facts upon which they supervene.

12 To forestall misunderstandings, these authors do not claim that the problem of arbitrary identification could be solved via the arbitrary reference strategy, for they are mainly concerned with arbitrary reference in instantial reasoning. So, I am extending the view to the case of proposition and numerical terms. 
13 Again, I shall here take issue solely with the answer to the metasemantic question. So, I won't be focusing on the second premise of this answer to the epistemic question.

14 The Acts of Choice Semantics could be further developed in two different directions. On the one hand, one might interpret such semantics in pretence-theoretic/fictionalist terms. That is to say, we can pretend that agents are acquainted with the relevant abstract objects and choose them as the referents of number and proposition terms. However, this fictionalist interpretation does not give rise to a realist solution to the problem of arbitrary identification. On the other hand, one might conceive of our practice of using proposition and numerical terms as bringing into the existence the very referents of such terms. This would make the referents of proposition and numerical terms akin to abstract artifacts. Even though this view can deliver a realist-friendly picture of the reference-fixing mechanism of the relevant expressions, it is not faithful to the reductionist approach, for a new category of objects, i.e. abstract artifacts, would have to be introduced in our ontological inventory. Thanks to François Recanati for discussion of this point.

15 See Hilbert and Bernays (1939), Slater (1988).

16 As a consequence, while Russell's $\imath$-term is an incomplete symbol, Hilbert's $\varepsilon$-term can be taken to play the role of what Russell calls logically proper names. This is why, in the Hilbertian reading, definite descriptions have a meaning independently of their use in a sentence.

17 I will henceforth focus, for the sake of simplicity, on proposition terms. But the following considerations carry over to numerical terms.

18 Following Nelson Goodman's definition, let us say that an object is grue if and only if the object is either (a) green and has been observed before now, or (b) blue, and has not been observed before now.

19 See also Cameron (2010) and Sider (2011) for other extensions of naturalness beyond properties.

20 The same consideration applies, mutatis mutandis, to numbers-naming expressions. That is to say, the working mathematician does not care whether ' 2 ' picks out a Zermelo set or a von Neumann set: all that matters is that the chosen entity is the next to the next to the least element of the chosen $\omega$-sequence.

21 Let me mention another option, though. According to Cameron (2010), it is possible to maintain that the ordering of naturalness facts is ontically indeterminate without jeopardising a realist semantics. Cameron develops the view with respect to vague predicates, but his main idea can be applied to our case as follows: the semantic fact that a given proposition-naming expression picks out a given entity is determined by use facts plus naturalness facts. While use facts are ontically determinate, naturalness facts are not. That is to say, the world leaves it unsettled whether a choice function is more natural than another.

22 A similar worry might be raised in relation to proposition terms. The considerations that follow are meant to apply to them as well.

23 See Moltmann (2013) for an extensive defence of this point.

24 See Jeshion (2015). 